\title{
Sore Throat Treatment Guidelines are Fanning the Flames of Antimicrobial Resistance
}

\author{
Interviewee: \\ Martin Duerden \\ University of Cardiff, Cardiff, UK \\ Disclosure: \\ Dr Duerden is a member of the Global Respiratory Infection Partnership (GRIP), \\ which receives an educational grant from Reckitt Benckiser (RB). \\ Support: \\ The publication of this article was funded by Reckitt Benckiser (RB) Group. \\ Acknowledgements: \\ Medical writing assistance was provided by Amanda Barrell, Brighton, UK. \\ Disclaimer: \\ The opinions expressed in this article belong solely to the named interviewee. \\ Citation: \\ EMJ Respir. 2020;DOI/10.33590/emjrespir/200914.
}

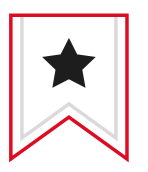

\section{Interview Summary}

Sore throat represents a significant yet under-recognised battle in the war against antimicrobial resistance (AMR). It is one of the most common reasons people visit a doctor and approximately $60 \%$ walk away with a prescription for antibiotics. ${ }^{1}$ However, studies have indicated that $70-95 \%$ of all cases are viral ${ }^{2}$ and most patients would be better served with symptom relief.

A systematic review of global sore throat management guidelines by members of the Global Respiratory Infection Partnership (GRIP) suggested the problem could be rooted in a focus on serious, yet increasingly rare, conditions, such as quinsy and acute rheumatic fever (ARF). While ARF can be a dangerous complication of Group A streptococci (GAS), which are identified in $15-30 \%$ of sore throat cases, the incidence of ARF is exceedingly rare in most parts of the world. ${ }^{3}$ All but one of the 36 identified guideline documents discussed antibiotic therapy and less than two-thirds advocated the use of laboratory tests to confirm GAS. Just 50\% gave advice on symptom relief, which evidence suggests is the most appropriate approach in most cases. ${ }^{4}$

Dr Martin Duerden, lecturer in therapeutics and prescribing at Cardiff University, Cardiff, UK, member of the GRIP, recently retired general practitioner, and co-author of the review, believes countries should re-evaluate their guidelines.

In this interview, Dr Duerden talks about the role of fit-for-purpose sore throat guidelines in antimicrobial stewardship, the importance of appropriate symptom relief, and how coronavirus disease (COVID-19) could represent an opportunity for change.

\section{ANTIMICROBIAL STEWARDSHIP AND SORE THROATS}

More than one-half of the people who visit a healthcare professional with sore throat, one of the most common health complaints globally, are prescribed antibiotics. ${ }^{1}$ Historically this approach was employed, among other reasons, to avoid ARF, which was much more common in the 1950 s and 1960s. This is a complication of GAS infection that can lead to rheumatic heart disease, mortality, and morbidity. While it is still 
seen in some low-to-middle-income countries in Africa, Southeast Asia, and the Western Pacific, ARF is actually exceedingly rare elsewhere. ${ }^{4}$ The overwhelming majority of sore throats are viral in origin, said Dr Duerden, adding that antibiotics are often not even required in the $15-30 \%$ of cases in which GAS is identified. The over-prescription of antibiotics for sore throat, which is better treated with symptom relief such as paracetamol, nonsteroidal anti-inflammatory drugs (NSAID), lozenges, and sprays, is "really quite concerning," commented Dr Duerden.

"We are seeing increasing resistance to antibiotics and are finding severe infections, such as septicaemia, difficult to treat. We now know there's really a large number of deaths associated with resistant bacteria that don't respond to standard therapy." ${ }^{5}$ Continuing, Dr Duerden emphasised that: "We have not had a new class of antibiotics for about 40 years and we are running out of them, the use of antibiotics for sore throat is actually stoking up the problem."

\section{GUIDELINE REVIEW}

To check the consistency of diagnostic and treatment criteria for sore throat, Coutinho et al. $^{3}$ carried out a multilingual, multi-region, systematic literature search that identified 36 treatment guidelines for sore throat from 26 countries. $^{3}$ All but one provided criteria for starting antibiotic therapy as a first-line treatment, but fewer than two-thirds advocated the use of laboratory tests to diagnose GAS prior to initiating therapy. Importantly, only one-half discussed symptom relief, which $\mathrm{Dr}$ Duerden described as the mainstay of treatment in most cases. ${ }^{3}$

Combined with the right diagnostic tests, antibiotics may be an important part of ARF containment strategies in high-risk areas. However, the approach is irrelevant in the many countries where ARF is exceedingly rare, $\mathrm{Dr}$ Duerden explained.

The study also highlighted worrying regional differences between the guidelines. Discrepancies were explained by historical, rather than current, incidences of ARF for example, or by guidelines being adopted from neighbouring countries with no adaptation for local circumstances. Guidelines may be bypassed altogether in countries where antibiotics can be purchased without a prescription, such as in Southeast Asia, and are unavailable in some regions, such as parts of Africa. The absence of guidance in Nigeria, for example, means most people presenting with sore throat are prescribed antibiotics as a matter of course.

All of these factors result in a significant volume of antibiotics being inappropriately prescribed and taken. "We're in a situation where we must reduce unnecessary antibiotic use. It is critical to make sure that we have clear ideas and structures around the processes of care, and that's particularly so for sore throats," emphasised Dr Duerden.

Rather than promoting the use of antibiotics, sore throat guidelines should ensure people are recommended symptom relief, Dr Duerden explained, adding that the review found extremely limited advice in this regard. Just 20 of the 36 guidelines recommended symptom relief such as paracetamol or NSAID, and only four of these mentioned topical treatments such as lozenges, gargles, or sprays, which evidence has suggested can treat symptoms much more effectively than antibiotics. "The most effective way of treating sore throat in nearly all parts of the world is providing symptom relief. Antibiotics have a very small part to play in terms of affecting the outcome of the infection," highlighted Dr Duerden.

Global approaches to the treatment of sore throat represent an international threat to antimicrobial stewardship, yet Dr Duerden fears the issue is not taken seriously. "Sore throats have been treated this way for 30 or 40 years and people don't think it's particularly important. They don't see it as being part of the problem," he said.

\section{DEVELOPING THE GUIDELINES OF THE FUTURE}

AMR is an issue receiving international oversight from bodies such as the World Health Organization (WHO). Focussing on the appropriate treatment of sore throat, Dr Duerden suggested, could represent a significant opportunity to make a difference. To this end, the GRIP has been encouraging countries to re-evaluate their guidelines. 
"If complications of GAS are a particular problem in your area, you will want to reduce infection and transmission. For most parts of the world, complications from sore throat are astonishingly rare and really what we're talking about is reducing symptoms," said Dr Duerden. "One possibility is developing or emulating high-quality guidelines which cover the essentials but are configured to local needs."

Guidelines should include a clear description of the disease, the diagnosis pathway, and necessary tests, as well as instructions on using symptom scores, which may indicate a more severe infection, he noted. Throat swab cultures are the gold standard for diagnosis of GAS and some countries advocate near-patient 'rapid antigen' testing. Where antibiotics are indicated, the guideline should explain the reasons, and set out the medication class and duration of treatment. "We also need to make sure people know the 'red flags', or the things likely to indicate severe infection, such as impaired swallowing, and when people should return if their symptoms persist," said Dr Duerden.

Critically, guidelines should advise on symptom relief, including paracetamol, NSAID, and topical treatments, such as lozenges, sprays, or gargles. Not only are these much more effective than antibiotics, they do not contribute to AMR, Dr Duerden explained.
Patient education is another consideration. Past experience often leads people with sore throat to ask their healthcare professional for antibiotics. Dr Duerden remarked: "We can educate around how sore throats are generally self-limiting, that you don't need to see a doctor, and that you can go to your pharmacist and take simple, symptomatic treatment."

\section{THE COVID-19 OPPORTUNITY}

The emergence of severe acute respiratory syndrome coronavirus-2 (SARS-CoV-2) could represent an opportunity to correct guideline omissions, avoid antibiotic overuse, and ensure people have access to evidence-based symptomatic treatment, said Dr Duerden. "I think COVID-19 is going to change things," he said. "Obviously, sore throat is a symptom of COVID-19. In the future, it's possible that when we see people with sore throat, we may need to do swabs or tests for coronavirus, and we need to think about how that gets built into guidelines."

"It's early days and we're not entirely sure how COVID-19 will pan out, but we need to be thinking about how we incorporate it into assessment for sore throat," he concluded.

\section{References}

1. Gulliford MC et al. Continued high rates of antibiotic prescribing to adults with respiratory tract infection: survey of 568 UK general practices. BMJ Open. 2014;4:e006245.

2. Worrall GJ. Acute sore throat. Can Fam Physician. 2007;53(11):1961-2.
3. Coutinho $\mathrm{G}$ et al. Major global discrepancies in guidelines for sore throat (acute pharyngitis). Poster 21400. ERS International Congress, virtual, 7-9 September, 2020.

4. Watkins DA et al. Global, regional, and national burden of rheumatic heart disease, 1990-2015. N Engl J
Med. 2017;377(8):713-22.

5. Cassini A et al. Attributable deaths and disability-adjusted life-years caused by infections with antibioticresistant bacteria in the $\mathrm{EU}$ and the European Economic Area in 2015: a population-level modelling analysis. Lancet Infect Dis. 2019;19(1):56-66. 